regard that the companion to HD114762 is probably well within the brown dwarf mass range. Planetary formation is thought to follow the creation of a protoplanetary disk during the formation of solar-type stars. There is no indication that a massive body such as the companion to HD114762, either rocky or gaseous, would form in this way in such close proximity to a solar-type primary. The significance of the companion of HD114762 for planetary formation is then solely exclusionary - one more solar-type star which probably does not have planets. The results of Campbell et al. ${ }^{3}$ are more interesting in this regard. Although they found no brown dwarfs, their sensitivity to low-amplitude velocity variations was sufficient to penetrate the regime of planetary mass detection (about 1-10 Jupiter masses). They identified several candidate systems. Extensive follow-up observations will be needed adequately to test the reality of these systems.

For the present, it is exciting to see positive results in the search for brown dwarfs obtained from a relatively small sample. It is promising for the future that this detection was achieved by straight-forward instrumental means on telescopes of moderate aperture. Uncertainties in the interpretation of results vis-a-vis the galactic-disk mass problem may be ameliorated by further observational work on the statistical properties of binary stars and by computational work with more generalized initial conditions. Those of us who search by other means can only encourage the radial-velocity observers, keeping in mind that well-defined and thorough programmes on carefully selected samples are the most likely to produce statistically meaningful results.

Ron Probst is at the Kitt Peak National Observatory, 950 North Cherry Street, Arizona 85719, USA.

\section{Latham. D.W. et al Nature 339, 3840 (1989)}

2. Marcy, G.W. \& Benitz, K.J. Astrophys. J. (in the press)

3. Campbell, B., Walker, G.A. \& Yang, S. Astrophys. J. 331 902-921 (1988).

4. Bahcall, J. N. Astrophys. J. 287, 926-944 (1984).

5. Liebert, J. \& Probst, R.G. A. Rev. Astron. Astrophys. 25, 473-519 (1987).

6. Zuckerman, B. \& Becklin, E.E. Nature 330, 138 (1987)

7. Forrest, W.J. et al. Astrophys. J. 330. L119-L123 (1988)

8. Abt, H.A. \& Levy, S.G. Astrophys. J. Suppl. 30, 273-306 (1976).

9. Boss, A.P. Comments Astrophys. 12, 180-190 (1988). 10. Boss, A.P. Astrophys. J. 319, 149-161 (1987).

\title{
Communities oceans apart?
}

\section{Stuart L. Pimm}

MANY ecologists can drive to their study sites - land, lakes and the intertidal. They often choose to study the behaviour, population dynamics and interspecific interactions of organisms experimentally on plots of 1-10 square metres. They receive training in mathematics and statistics and read a distinct set of journals. Biological oceanographers, on the other hand, sample across vast tracts of ocean from boats, and document the physical and chemical effects on biomass for which their training prepares them. At a recent meeting*, terrestrial, aquatic, intertidal and oceanic ecologists considered the similarities and differences of their systems and methods. They generally failed to agree, and my list is not a consensus either. There was unanimity, however, that the different views are an essential ingredient in solving some critically important ecological problems.

The comparative approach is essential in ecology, where many ideas cannot be tested experimentally. Comparisons of the number of species in marine and terrestrial systems provide one example that illustrates the strengths and weaknesses of such broad contrasts. The total number of species on land may be much greater than in the oceans (J. McGowan, Scripps Institute of Oceanography). But

\footnotetext{
* A Comparison of Marine and Terrestrial Ecosystems workshop held in Santa Fe, New Mexico, 14-16 March 1989. Organized by John Steele.
}

the numbers of species in poorly known tropical forests may only seem to be greater than in marine benthic communities because the latter are even less well known. Even if the difference is real, it could be explained because terrestrial communities are dominated by the excessively diverse insects, which are almost entirely absent from marine communities $(\mathrm{H}$. Caswell, Woods Hole Oceanographic Institute). Do insects have particular features allowing this diversity, or does it result from the terrestrial environment? Suppose that the difference results from the environment. Terrestrial diversity increases with increasing environmental patchiness because patches of different sizes (gaps in a forest, say) have their own idiosyncratic species. Satellite imagery shows that the physical features of the ocean are very patchy (J. Steele, Woods Hole Oceanographic Institute). Yet the cold water rings in the turbulent flow of an oceanic current may contain species common to cold water rather than species unique to the patches. What does this say about the role of movement and dispersal in driving species richness? Dispersal processes on land and in the sea are likely to be very different.

Differences in population dynamics may be the least contentious comparison. Ocean food chains have at their base phytoplankton with very short lifetimes and large, top predators with long life- times. On land, very long-lived trees and shrubs or slowly decomposing organic matter are at the base of the chain, with shorter-lived top predators and the shortest lived species sandwiched in the middle. (Of course, intertidal, benthic and coral-reef habitats are more like terrestrial habitats in this respect than the open oceans. A recurrent theme of the meeting was that the greatest differences are between planktonic and anchored communities.) Food-chain models show that the different patterns of longevity have a profound effect on community dynamics (R. Wiegert, University of Georgia). Given this difference in dynamics, it is perhaps surprising that many characteristics of food chains and food webs are so similar in terrestrial, aquatic and marine habitats (J. Cohen, Rockefeller University).

As significant as the differences in the systems studied is the difference in approaches and methods imposed by the different means of reaching study sites. No group is likely to export its ideas and methods to the other without the other seeing the potential profit in importing ideas (S. Levin, Cornell University). Nowhere is the importance of this more apparent than in considering the consequences of global climatic changes. The important questions of scale - for how long and how far physical variables are essentially unchanged - are commonplace in the marine literature yet are rarely addressed explicitly in the terrestrial literature (Levin). The characteristic spatial scales of physical variables in the ocean and atmosphere are the stuff of undergraduate lectures in oceanography, yet a review of these ideas (T. Powell, University of California, Davis) was news to many terrestrial ecologists. In contrast, an evolutionary perspective and details of behaviour, population dynamics and community interactions are probably better known on land. Yet what is critically important is how these biological details follow or resist the changes in physical variables, and this coupling has never been adequately studied.

Understanding how physical changes affect detailed biological interactions will come from hitherto unlikely alliances of ecologists. How should such alliances be encouraged? There are many places for ecologists to pursue field work, and there are summer courses galore to train students in practical skills. Yet there is no place for either researchers or students to think collectively or to analyse data. The pressing problems of the loss of biological diversity and environmental change suggest that this omission must soon be rectified.

Stuart L. Pimm is in the Department of Zoology and Graduate Program in Ecology. The University of Tennessee, Knoxville, Tennessee 37996, USA. 